\title{
Microwave Reflection Tomographic Array for Damage Detection of Civil Structures
}

\author{
Yoo Jin Kim, Luis Jofre, Member, IEEE, Franco De Flaviis, Member, IEEE, and Maria Q. Feng
}

\begin{abstract}
Microwave tomographic imaging technology using a bifocusing operator has been developed in order to detect the internal voids/objects inside concrete structures. The imaging system consists of several cylindrical or planar array antennas for transmitting and receiving signals, and a numerical focusing operator is applied to the external signals both in transmitting and in receiving fields. An imaging algorithm using numerical focusing operator was developed, which allows the recovery of a two-dimensional object from its scattered field. Numerical simulation demonstrated that a subsurface image can be successfully reconstructed by using the proposed tomographic imaging technology. For the experimental verification, a prototype planar antenna array was fabricated and tested on a concrete specimen.
\end{abstract}

Index Terms-Damage detection, imaging, reflection tomography.

\section{INTRODUCTION}

$\mathbf{N}$ ONDESTRUCTIVE assessment of concrete structures, currently, heavily relies on visual inspections, which apparently have some limitations. Majority of highway bridges are concrete bridges, and invisible damage such as voids and cracks inside concrete and debonding between rebars and concrete caused by corrosions and earthquakes is of significant concern.

In previous work [1]-[4], the authors developed an electromagnetic imaging technology using surface-focused microwave. This was developed to assess the bonding condition of fiber reinforced polymer (FRP)-jacketed concrete structures. The experimental results successfully detected the area and the location of the voids. Accurate information about the void's depth, however, cannot be obtained easily because the correlation of the depth and the measured signal has to be experimentally determined. Apparently, it is not easy for all possible damage cases.

In this paper, a subsurface-focused microwave imaging technology using transmitting and receiving arrays, as shown in Fig. 1, is developed, in order to assess damage within a concrete structural element far away from its surface and to obtain the depth information about damage. This technology can construct a microwave image, showing the information under the surface, thus it is possible to detect the depth of damage in concrete. Furthermore, this technology uses transmitting and receiving arrays with several antennas and the focusing point can be quickly adjusted by software without moving the hardware, which makes it possible to quickly measure a large area involving many points.

Manuscript received March 26, 2002; revised December 30, 2002. This work was supported by the National Science Foundation under Grants CMS-9812585 and CMS-9812856.

Digital Object Identifier 10.1109/TAP.2003.818786

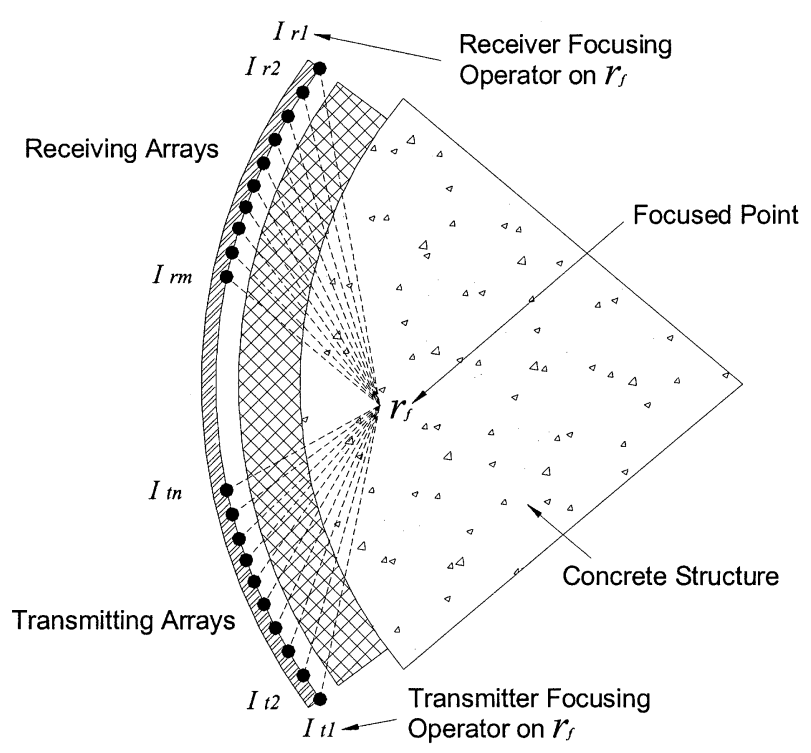

Fig. 1. Use of microwave reflection arrays to focus waves on subsurface point.

The proposed subsurface imaging technology uses an arrangement consisting of several cylindrical or planar array antennas for transmitting and receiving signals, and a numerical focusing operator is applied to the external signals both in transmitting and in receiving fields. First, this paper describes a numerical focusing procedure, which allows the recovery of a two-dimensional (2-D) tomographic image of an object from its scattered field. Second, some numerical simulations for the study of reconstruction parameters and the verification of reconstruction algorithm are presented. Finally, experimental verification through the tests on the concrete blocks demonstrated that the subsurface focused imaging technology, using the proposed antenna array, can successfully detect the voids and defects inside the concrete structure.

\section{RECONSTRUCTION AlgORITHM}

\section{A. Analytical Formulation}

The measurement geometry, shown in Fig. 2, uses $N_{n} \times N_{m}$ elements, $N_{n}$ forming a cylindrical transmitting array and $N_{m}$ forming a cylindrical receiving array. A $N_{n} \times N_{m}$ measurement matrix can be obtained as follows: for every selected transmitting element, the receiving array is scanned obtaining an $N_{m}$-measurement column, then the procedure is repeated for the $N_{n}$ elements of the transmitting array. Due to the basic 2-D characteristic of the geometry under study, each element consists of a long vertical antenna array with a uniform current distribution. In practical terms, the length of the vertical antenna 


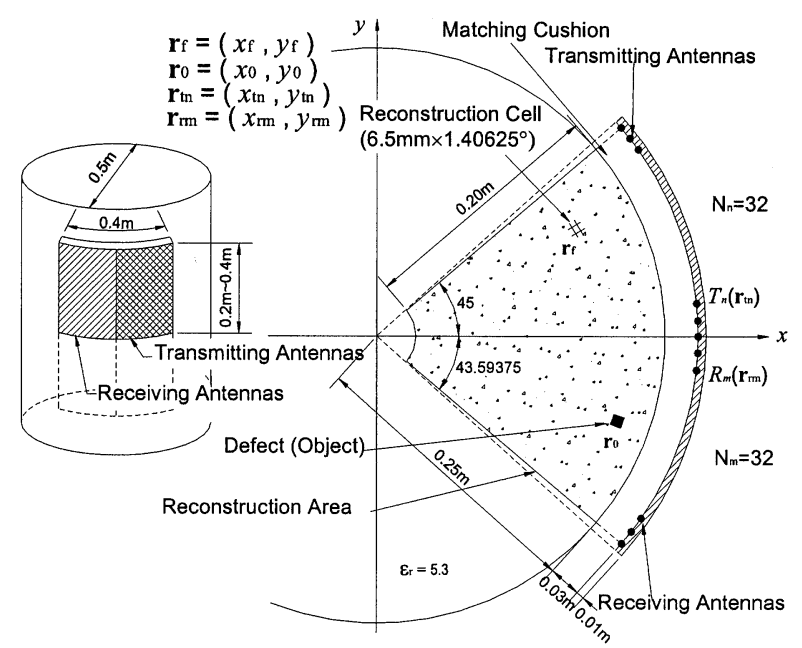

Fig. 2. Measurement geometry for two cylindrical transmitting and receiving arrays of 32 elements.

array has to be greater than the transversal dimension of the focusing area. The concrete was assumed to be homogeneous and the effects from aggregates size were not considered in this theoretical model.

Following the electromagnetic compensation principle, the illumination of an object induces an equivalent electric current distribution, $J_{e q}\left(x_{0}, y_{0}, z_{0}\right)$, and this distribution makes an electromagnetic image of the object in image reconstruction [5], [6]. The reconstruction algorithm forms every image point by means of the synthesis of two focused arrays (transmitting and receiving arrays), i.e., all the elements of both arrays are weighted by focusing operator so as to be focused on a unique object point. This can be achieved by numerical treatment of the measurement matrix.

The focusing operator can be obtained by taking an inverse of the field induced by a current line. It is well known that the electric fields of the infinite electric line source are proportional to a Hankel function of the second kind whose argument is proportional to the distance from the source to the observation point. Therefore, the incident field at $\mathbf{r}_{i}\left(x_{i}, y_{i}\right)$ when focusing on every transmitting point $\mathbf{r}_{t n}\left(x_{t n}, y_{t n}\right)$ on the reconstructing point $\mathbf{r}_{f}\left(x_{f}, y_{f}\right)$ can be expressed as

$$
E_{i}\left(x_{i}, y_{i}\right)=\sum_{n=1}^{N_{n}} I_{T n}\left(x_{f}, y_{f}\right) \cdot H_{0}^{(2)}\left(k_{e}\left|\mathbf{r}_{t n}-\mathbf{r}_{i}\right|\right)
$$

where $I_{T n}\left(x_{f}, y_{f}\right)$, the focusing operator, is given by

$$
I_{T n}\left(x_{f}, y_{f}\right)=\frac{1}{H_{0}^{(2)}\left(k_{e}\left|\mathbf{r}_{t n}-\mathbf{r}_{f}\right|\right)}
$$

and $k_{e}$ is wavenumber in concrete $\left(\varepsilon_{r}=5.3\right)$.

Scattered field measured at $\mathbf{r}_{r m}\left(x_{r m}, y_{r m}\right)$ of a defect (object) placed at $\mathbf{r}_{0}\left(x_{0}, y_{0}\right)$ is

$$
E_{s}\left(x_{r m}, y_{r m}\right)=E_{i}\left(x_{0}, y_{0}\right) \cdot I_{o b j} \cdot H_{0}^{(2)}\left(k_{e}\left|\mathbf{r}_{r m}-\mathbf{r}_{0}\right|\right)
$$

where $I_{o b j}$ is a constant for every object containing its electromagnetic macroscopic characteristics.

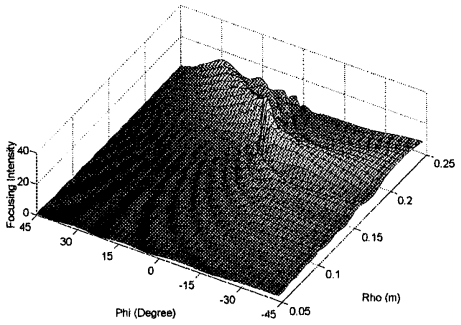

(a)

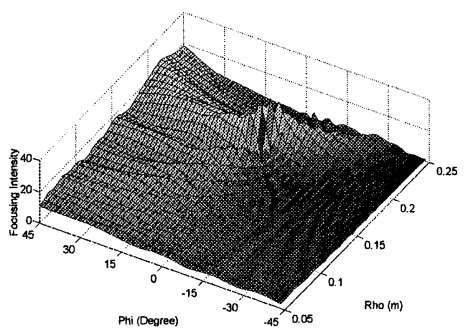

(b)

Fig. 3. Focusing intensity in concrete (focused point: $\rho=0.20 \mathrm{~m}, \phi=0^{\circ}$ ). (a) Transmitting intensity. (b) Receiving intensity.

When focusing back the received field at $\mathbf{r}_{r m}\left(x_{r m}, y_{r m}\right)$ on the point of interest $\mathbf{r}_{f}\left(x_{f}, y_{f}\right)$, the electromagnetic image of $E_{f}\left(x_{f}, y_{f}\right)$ at $\mathbf{r}_{f}\left(x_{f}, y_{f}\right)$ can be expressed as

$$
E_{f}\left(x_{f}, y_{f}\right)=\sum_{m=1}^{N_{m}} E_{s}\left(x_{r m}, y_{r m}\right) \cdot I_{R m}\left(x_{f}, y_{f}\right)
$$

where $I_{R m}\left(x_{f}, y_{f}\right)$, the focusing operator, is given by

$$
I_{R m}\left(x_{f}, y_{f}\right)=\frac{1}{H_{0}^{(2)}\left(k_{e}\left|\mathbf{r}_{r m}-\mathbf{r}_{f}\right|\right)} .
$$

Finally, the entire process can be grouped as follows:

$$
\begin{aligned}
& E_{f}\left(x_{f}, y_{f}\right)=\left[\begin{array}{llll}
I_{T 1} & I_{T 2} & \cdots & I_{T n}
\end{array}\right] \\
& \cdot\left[\begin{array}{cccc}
E_{s, T 1 R 1} & E_{s, T 1 R 2} & \cdots & E_{s, T 1 R m} \\
E_{s, T 2 R 1} & E_{s, T 2 R 2} & \cdots & E_{s, T 2 R m} \\
\vdots & \vdots & \ddots & \vdots \\
E_{s, T n R 1} & E_{s, T n R 2} & \cdots & E_{s, T n R m}
\end{array}\right] \cdot\left[\begin{array}{c}
I_{R 1} \\
I_{R 2} \\
\vdots \\
I_{R m}
\end{array}\right]
\end{aligned}
$$

\section{B. Reconstruction Parameters}

The formulation derived in the previous section has been applied to the case of two cylindrical arrays of 32 antennas each, at a frequency of $10.0 \mathrm{GHz}\left(\lambda_{0}=3.0 \mathrm{~cm}, \lambda_{e}=1.3 \mathrm{~cm}\right.$ ). Fig. $3(\mathrm{a})$ and (b), respectively, show the focusing intensity of transmitting and receiving signals in concrete when focused at the point of $\left(\rho=0.20 \mathrm{~m}, \phi=0^{\circ}\right)$, which is $(x=0.20 \mathrm{~m}, y=0 \mathrm{~m})$ in rectangular coordinates. The focusing intensity reaches the maximum value (32) at the focused point. This value is consistent with the number of transmitting and receiving antennas.

In order to study the focusing capability of the system at different distances and view angles, nine point-like objects were placed at the boundary and the central landmark point of the reconstructing 2-D cross section. The results, as shown in Fig. 4, show good uniformity in the focusing intensity levels, which is 


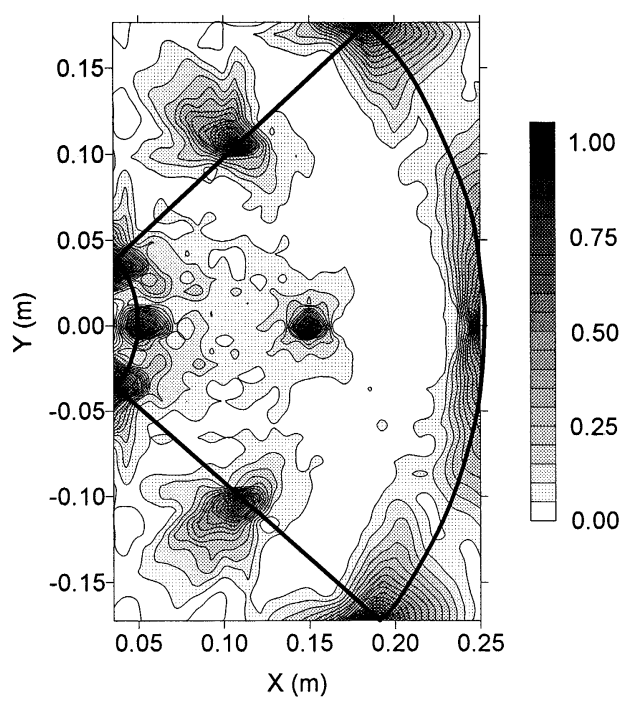

(a)

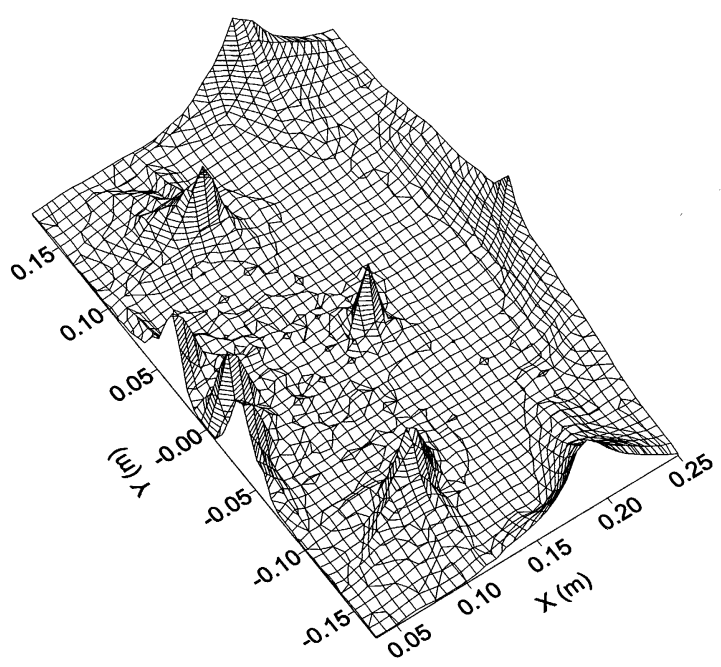

(b)

Fig. 4. Image of nine point-like objects (Impulse response of the system). (a) $x-y$ plane image (normalized unit from 0 to 1). (b) 3-D image.

normalized from 0 to 1 , at the 9 points. The impulsive shape of the focusing intensity at the focused point suggests a very good behavior of the reconstruction algorithm.

Finally, simulations using numerical measurements were conducted in order to verify the resolution capabilities of the system. As shown in Fig. 5, the results demonstrate that the system, due to the use of bifocusing (focusing both in the transmitting and receiving arrays), is able to achieve a resolution in the order of the wavelength, both in transversal and in the longitudinal directions, in the dielectric medium. This clearly improves the resolution of a conventional system using mono-focusing (focusing the receiving array only) [7].

\section{ANTENNA ARRAY DESIGN}

\section{A. Design of Slot Antenna Arrays}

Based on the electrical and geometrical parameters of the concrete specimen to be inspected, an illuminating frequency of $5.2 \mathrm{GHz}$ was chosen as it represents a reasonable tradeoff

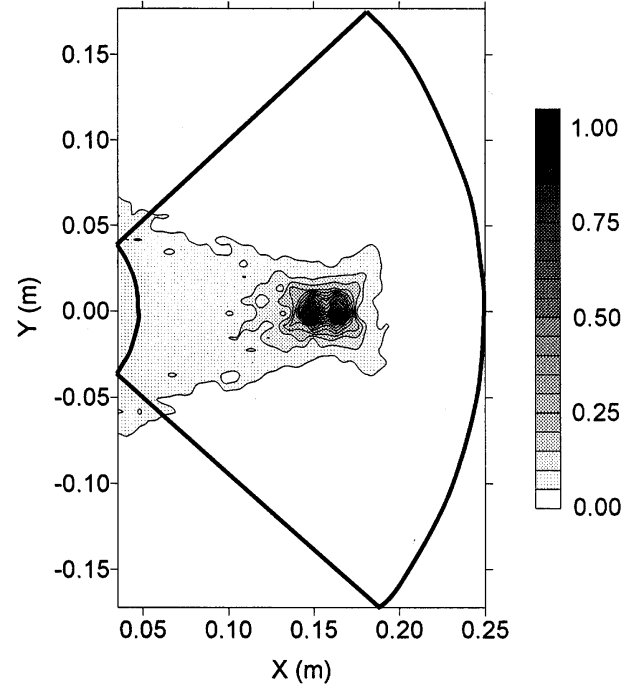

(a)

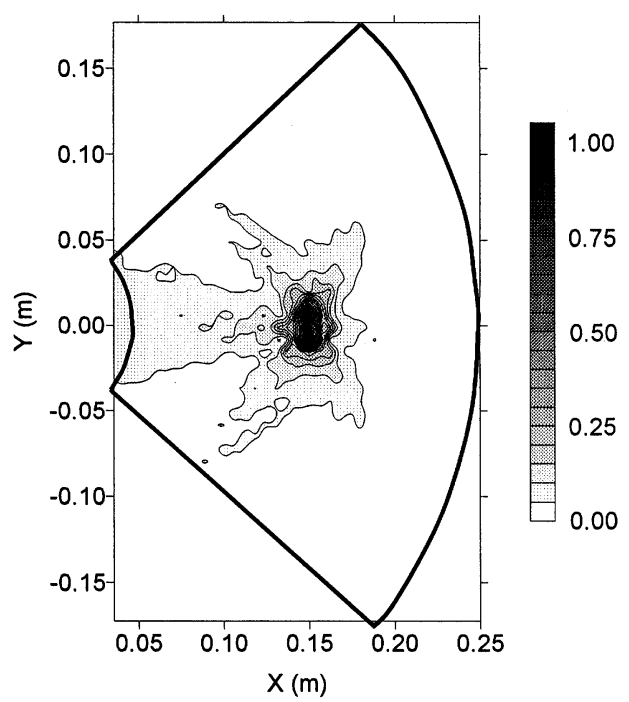

(b)

Fig. 5. Reconstructed images of two point-like objects. (Resolution of the system in normalized units from 0 to 1). (a) Transverse distance of $0.013 \mathrm{~m}$. (b) Longitudinal distance of $0.013 \mathrm{~m}$.

between the signal attenuation and the image resolution. The resolution can be $2.5 \mathrm{~cm}$ at $5.2 \mathrm{GHz}$, which is obtained from the wavelength $\left(\lambda_{e}\right)$ in concrete. Also, the 5.2-GHz frequency was chosen as an adequate 1:2 or 1:4 scale model for the geometries and wavelengths of the real columns and also to work with dimensional tolerances that facilitate the laboratory.

A microstrip slot antenna was chosen in order to be directly attached to the concrete surface or the matching cushion. The microstrip slot antenna has the advantage of being able to produce either bi-directional or unidirectional radiation patterns with a large bandwidth. The strip and slot combination offers an additional degree of freedom in the design of the microstrip antenna [8], [9].

At last, the geometry of the slot antenna was determined so as to obtain a wide bandwidth at the resonance frequency of 5.2 GHz. Fig. 6. illustrates the design based on the considerations described above. $64(8 \times 8)$ slots for each array and a dimension 


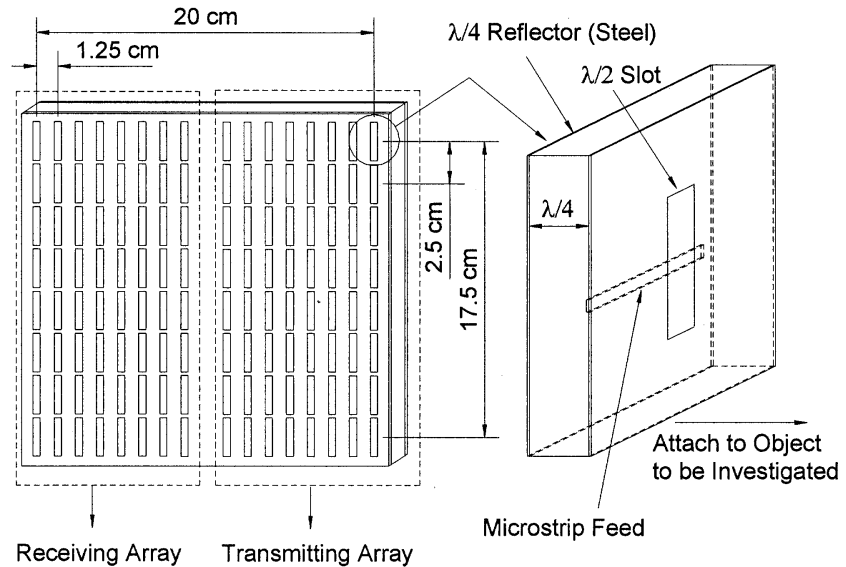

(a)

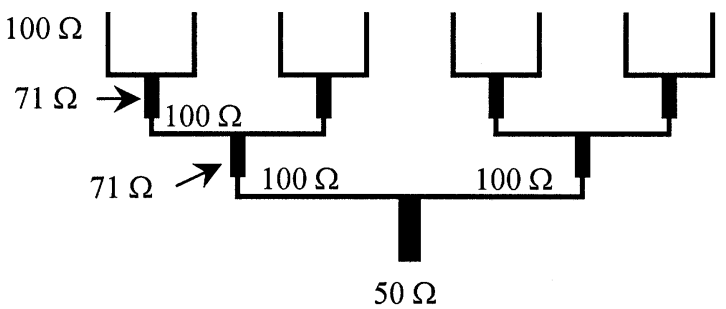

(b)

Fig. 6. Planar rectangular microwave antenna array. (a) Conceptual design of planar rectangular slot antenna array. (b) Eight elements linear array with corporate feed configuration.

of $20 \mathrm{~cm} \times 20 \mathrm{~cm}$ were selected as they represent a reasonable tradeoff between the resolution and the reconstructed area covered by the antenna array.

As shown in Fig. 6, the planar rectangular microwave antenna is composed of transmitting and receiving arrays, each consisting of $8 \times 8$ slot antennas. Each of these two planar $8 \times$ 8 arrays consists of a parallel fed eight-element uniform vertical array producing a tomographic focused slice perpendicular to the vertical axis of the structure, and an electronically switched eight-element horizontal array able to focus at a particular point inside the previous tomographic focused slice. The transmitting antenna array focuses the illuminating fields on a particular point inside the volume of investigation and the receiving array focuses the receiving beam on the same point. The antenna array is a sandwich structure with two metallic grounded substrates separated by a light foam layer. The grounded substrate close to the target concrete structure contains the radiating half-wavelength $(\lambda / 2)$ slots in the exterior ground plane and the $100 \Omega$ microstrip feeding line on the interior side. The second grounded plane, quarter-wavelength $(\lambda / 4)$ apart from the slot plane, acts as a reflector in order to produce unidirectional radiation toward the volume of investigation.

\section{B. Measurement Results}

The fabricated antenna array was placed on a concrete specimen with the slots facing the surface of the concrete, so that the wave radiates through the concrete. The reflection $\left(S_{i i}\right)$ and transmission parameters $\left(S_{i j}\right)$ were tested in order to investigate the matching condition and mutual coupling. Fig. 7. shows

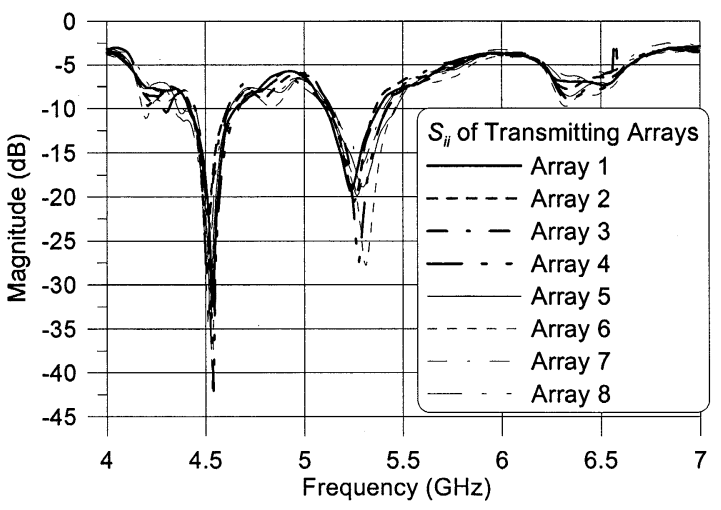

(a)

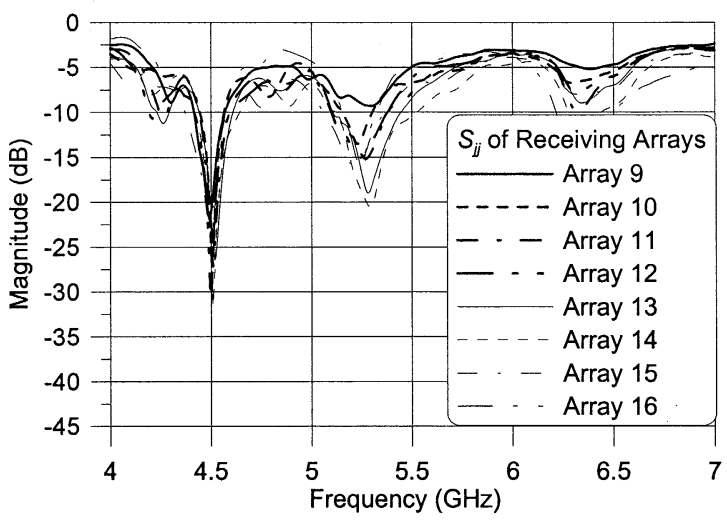

(b)

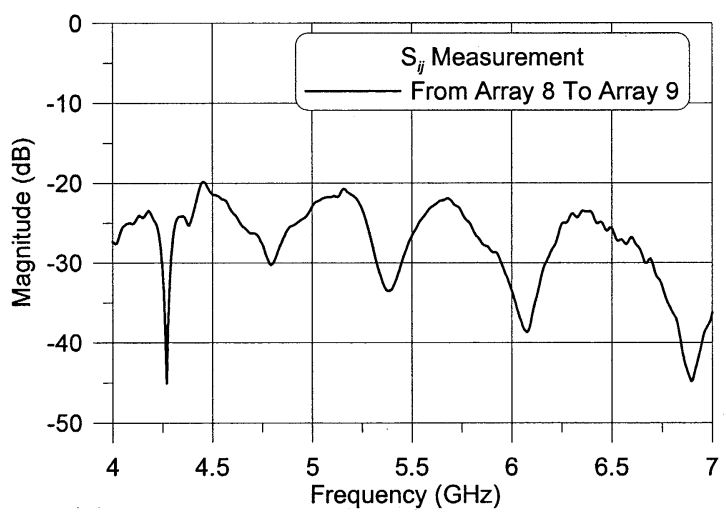

(c)

Fig. 7. Measurement results of $8 \times 16$ antenna array. (a) $S_{i i}$ measurement of transmitting array. (b) $S_{j j}$ measurement of receiving array. (c) Example of $S_{i j}$ measurement $\left(S_{89}\right)$.

a good radiation performance at the illuminating frequency, and that interference between the colateral elements is as low as -20 $\mathrm{dB}$.

\section{NUMERICAL SimULATION}

Numerical simulations were carried out to test the proposed algorithm for the image reconstruction of a concrete specimen with internal air voids and steel bars. The reconstruction parameters including effective focusing area were investigated. In total, seven cases were modeled with $5.2 \mathrm{GHz}$ as an illuminating frequency and the image of each case was reconstructed. Some of these numerical simulations were compared with the results 


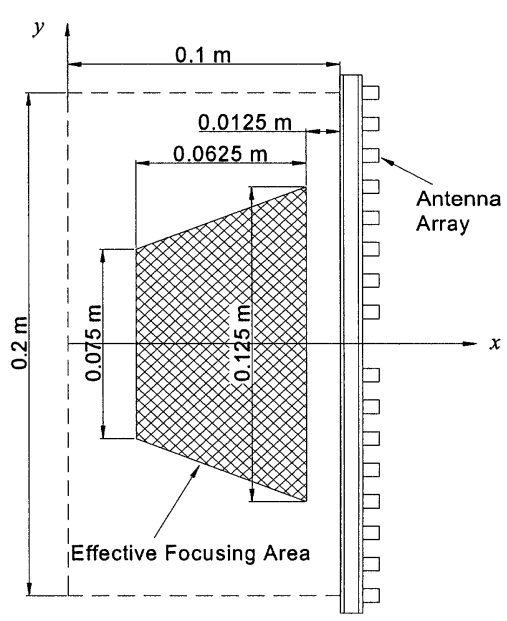

Fig. 8. Effective focusing area of planar rectangular antenna array.

with $10.0 \mathrm{GHz}$ as the illuminating frequency. This comparison showed that the resolution can be improved by using the higher illuminating frequency. For the simulation, planar rectangular antenna arrays were used with 8 transmitting/receiving antennas for the case of $5.2 \mathrm{GHz}$ and 16 transmitting/receiving antennas for the case of $10.0 \mathrm{GHz}$. For simplicity, only a 2-D rectangular geometry was considered for the investigation and only Y polarization of the antenna was used. Extension to 3-D can be easily obtained by using the spherical Green's function as a focusing operator instead of the Hankel function.

\section{A. Effective Focusing Area (EFA)}

Reconstruction parameters such as focusing intensity and impulse response of the system for the planar antenna array were investigated again, because the geometry of the fabricated antenna array and the frequency range are different from the cylindrical system used previously. Based on the results of a parametric study using point-like objects, the effective focusing area (EFA), in which the focusing operation is effective, was determined. In other words, the focusing intensity level and impulse response shape have a good uniformity in the effective focusing area. Also, the effective penetration depth is determined by the effective focusing area. The shape and size of the effective focusing area (penetration depth) strongly depend not only on the electrical size of the measuring line, but also on the arrangement of the transmitting and receiving elements, which means that the EFA of a planar antenna array differs from the one for circular antenna array. Through a series of simulation using point-like objects, in which the focusing intensity level and shape of impulse response at different locations were compared, the effective focusing area was determined as the shape of a trapezoid, as represented in Fig. 8.

\section{B. Simulation Results Using $5.2 \mathrm{GHz}$}

Based on the reconstruction parameters, especially the effective focusing area, numerical simulations were carried out. For the direct problem in which the antenna radiates a wave and receives the scattered field, a 3-D structure simulator, CST Microwave Studio, was used for measuring the transmission parameters $\left(S_{i j}\right)$ in each array. Totally, seven geometries were modeled with a 16-slot antenna. In the simulation, the background material was modeled as concrete so as to radiate the wave through the concrete. Open boundary conditions were applied at the top and the bottom of the geometry in order to generate absorbing conditions.

Using the $S_{i j}$ measurements from each array, the measurement matrix of (6) was assembled and the electromagnetic image of $E_{f}\left(x_{f}, y_{f}\right)$ at $\mathbf{r}_{f}\left(x_{f}, y_{f}\right)$ was obtained as presented previously.

All the objects used in the numerical simulation were located inside of the effective focusing area. The reconstructed images for each case are reported in Fig. 9 along with the model description and the reconstructed area.

In case 1, the air sphere was successfully reconstructed with exact location and size as represented in Fig. 9(a). The reconstructed area was limited to $5 \mathrm{~cm}$ in $x$ direction because the open boundary in CST Microwave Studio was set at the $y$-axis, from which the distance to the antenna array is $5 \mathrm{~cm}$. The reconstructed area in the $x$ direction for the other cases was also determined by the location of an open boundary $(10 \mathrm{~cm}$ in the $x$ direction).

Cases 2, 3, and 4 are similar with the ones in the parameter investigations in Section II, in which the system was able to achieve resolution of the order of a wavelength in the dielectric medium. Cases 2 and 3 show the transverse and longitudinal resolution of the system, respectively. The wavelength in concrete at $5.2 \mathrm{GHz}$ is about $2.5 \mathrm{~cm}$, which provides the resolution of the system of $2.5 \mathrm{~cm}$.

As represented in Fig. 9(b), the locations of two steel bars separated with transverse distance of $2.5 \mathrm{~cm}\left(\lambda_{e}\right)$ were detected apparently, although the resolution was not sufficient enough to reconstruct the exact shapes of the bars. Fig. 9(c) shows the reconstructed image of two steel bars placed at a longitudinal distance of $2.5 \mathrm{~cm}\left(\lambda_{e}\right)$. In this case, the two bars could be successfully identified, but the exact shapes could not be reconstructed due to the lack of resolution. From the results of cases 2 and 3, it was demonstrated that the resolution of the system with planar antenna array is of the order of a wavelength in concrete (2.5 $\mathrm{cm}$ ), which can identify the approximate shapes and locations.

In case 4, six steel bars were placed at the boundaries of the effective focusing area for the reconstructing of a 2-D cross section. The result, as shown in Fig. 9(d), demonstrated that the objects near the antenna arrays are more clearly identified rather than the ones at the farther boundaries of effective focusing area, which is marked by black line, from the antenna array.

In cases 5 and 6, a square and a rectangular air block were placed inside the concrete material, respectively. As shown in Fig. 9(e) and Fig. 9(f), the reconstructed image shows the exact location and the approximate size of the air blocks in both cases, although there was some noise. These two cases were investigated again at the higher frequency in order to demonstrate the improvement of resolution.

In case 7, a steel square bar and an air block were placed inside the concrete material. The difference in the dielectric constant between steel and concrete is much bigger than the one between air and concrete. For this reason the air void is more difficult to detect than the steel bar. As shown in Fig. 9(g), although the steel is located far from the antenna array, the main 


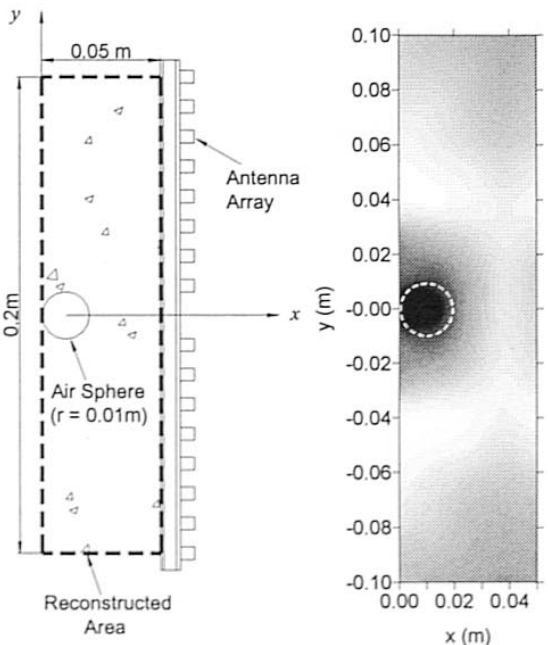

(a)

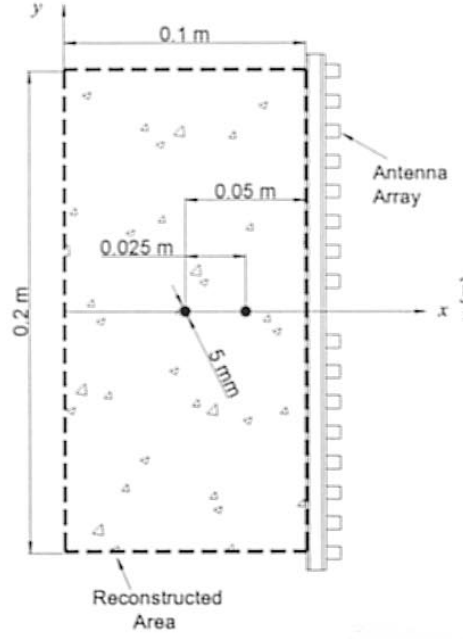

(c)

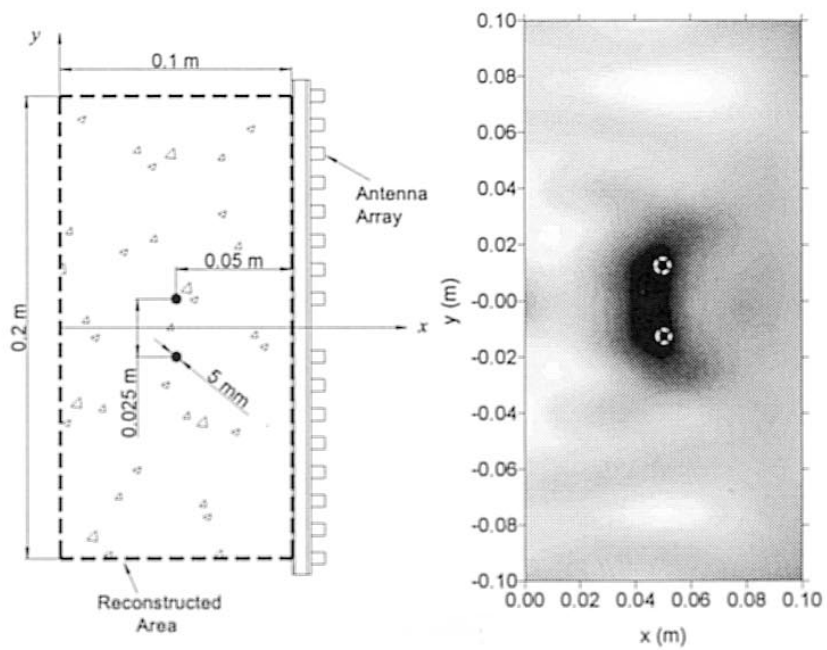

(b)

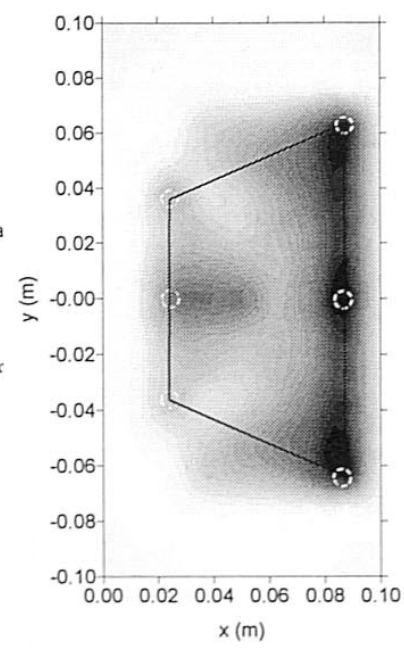

(d)

Fig. 9. Simulation results with descriptions: (a) Case 1, (b) Case 2, (c) Case 3, and (d) Case 4.

features are correctly located, including size and position, while for the air void only the location is detected.

Using the results of the numerical simulation, the efficiency of the image reconstruction algorithm was tested through different cases, including steel, air voids, or combinations of the above. Within the effective focusing area, the location and size of objects were successfully detected.

\section{Simulation Results Using $10.0 \mathrm{GHz}$}

In the antenna design, the illuminating frequency was determined as $5.2 \mathrm{GHz}$ as a good compromise between a set of different parameters, such as size, resolution, attenuation, and simplicity of prototype. For that reason, the resolution of the system, as shown in the previous simulation results, was limited to 2.5 $\mathrm{cm}$ and the exact shape of the object inside concrete could not be reconstructed. Better resolution, however, can be obtained by increasing the illuminating frequency.

In order to demonstrate the improvement of resolution using higher illuminating frequency, cases 5 and case 6 were investigated again with $10.0 \mathrm{GHz}$ as an illuminating frequency. For the numerical simulation, the planar rectangular antenna arrays with 16 transmitting and 16 receiving antennas were used. The results in Fig. 10 show that the resolution of the reconstructed image can be improved by increasing the illuminating frequency and that the exact shape and location of the voids can be reconstructed when using $10.0 \mathrm{GHz}$.

\section{EXPERIMENTAL IMPLEMENTATION}

\section{A. Experimental Setup and Calibration}

The effectiveness of the proposed subsurface imaging technology using the developed microwave antenna array was investigated through a series of experiments on a concrete panel and a concrete block. In the experiment, a concrete panel with a hole at the center and a rectangular concrete block were used. The concrete panel was first used for calibration (to be described). Then, a sphere hole was placed on one of the faces of the concrete panel to simulate damage. Two types of artificial voids were generated inside a concrete block with a dimension of $30 \mathrm{~cm} \times 30 \mathrm{~cm} \times 30 \mathrm{~cm}$. The first involved a square Styrofoam block of $2 \mathrm{~cm}$ on the side and the other involved a rectangular Styrofoam bar of $5 \mathrm{~cm} \times 2 \mathrm{~cm} \times 2 \mathrm{~cm}$. The Styrofoam 


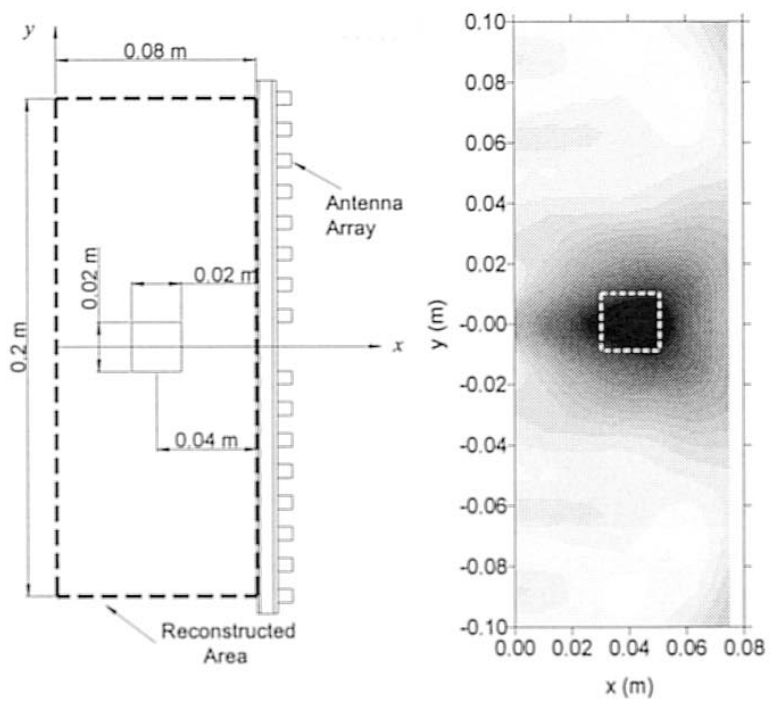

(e)

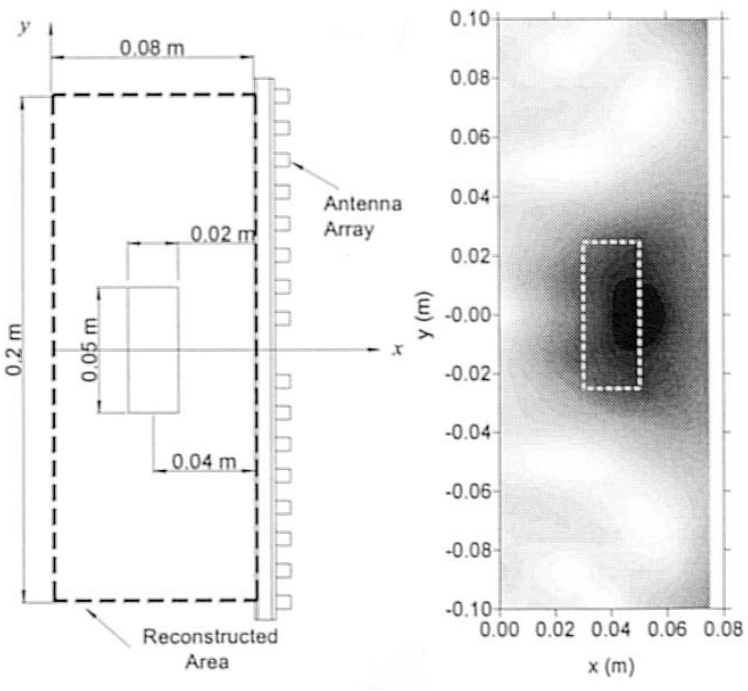

(f)
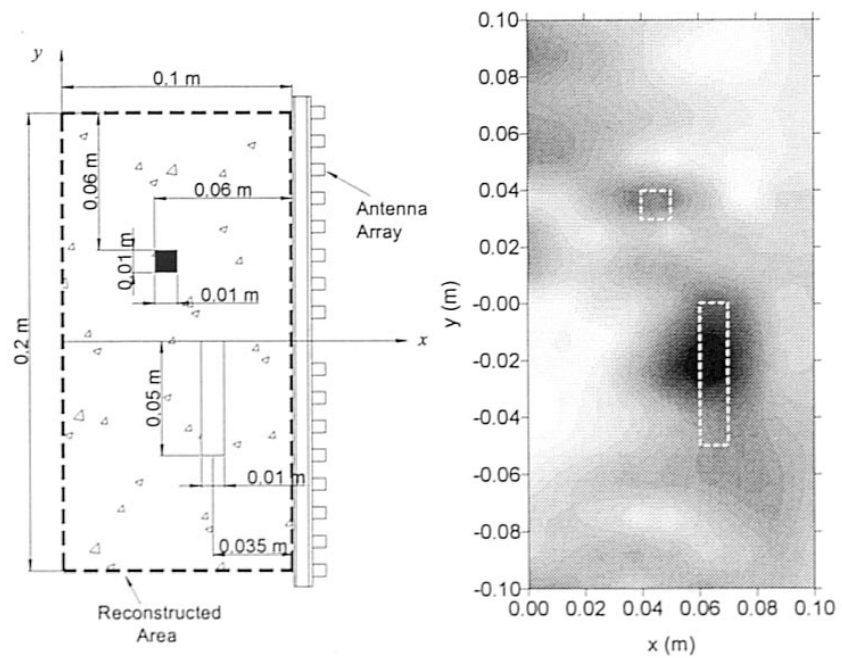

(g)

Fig. 9. (Continued.) Simulation results with descriptions: (e) Case 5, (f) Case 6, (g) Case 7.

block was inserted into the concrete during the pouring of concrete with the distance of $3 \mathrm{~cm}$ from the face of concrete to the face of Styrofoam in both cases. The samples using steel bars in the air were also prepared. All the experimental cases were described in Table I.

The experimental setup consisting of a network analyzer, a switch box, coaxial cables and antenna arrays is represented in Fig. 11. The network analyzer was used to evaluate the transmitted portion of signal through the medium $\left(S_{12}\right)$. The switch box is a RF network capable of controlling multiple antennas in the array and selecting them individually to perform $S_{12}$ measurements.

In order to remove the effect of wave reflections and loss in the coaxial cables and/or in the switch box from the measurement matrix, calibration with respect to each transmission measurement $\left(\mathrm{S}_{i j}^{(m e a)}\right)$ is needed. For the calibration purpose, a concrete panel without defect and steel plate were used as represented in Fig. 12.

Each transmission measurement, without all the effects from coaxial cables and switch box, which is from the transmitting array to the receiving one, is proportional to the zero-order Hankel function of the second kind, $H_{0}^{(2)}\left(k_{e, \text { conc }} d_{i j}\right)$, whose argument is proportional to the distance from the transmitting array to the receiving one, $d_{i j}[10]$. All the measurement effects from the coaxial cables and the switch box (calibration factor), therefore, can be calculated by dividing each transmission measurement of calibration $\left(\mathrm{S}_{i j}^{\left(c a l \_m e a\right)}\right)$ by the zero-order Hankel function of the second kind, and the calibrated transmission measurement $\left(\mathrm{S}_{i j}^{(c a l)}\right)$ can be obtained by dividing each transmission measurement $\left(\mathrm{S}_{i j}^{(m e a)}\right)$ by a calibration factor, as follows for the case of transmitting array $i$ and receiving array $j$.

$$
S_{i j}^{(c a l)}=\frac{S_{i j}^{(\text {mea })}}{\text { Cal_Factor }}
$$

where

$$
C a l_{-} f a c t o r=\frac{S_{i j}^{\left(c a l \_m e a\right)}}{H_{0}^{(2)}\left(k_{e, \text { conc }} d_{i j}\right)} .
$$

The amplitudes of the calibrated measurements in concrete without defects are proportional to the distance between the 


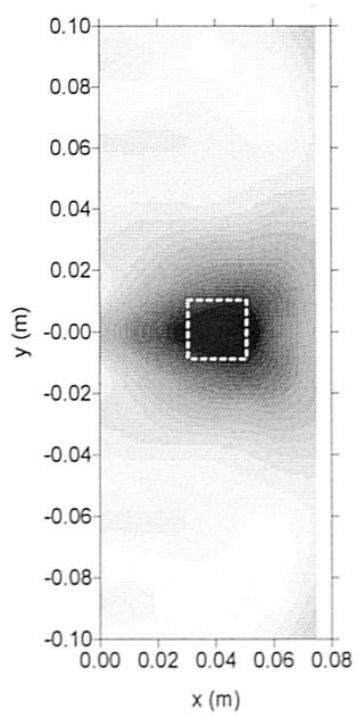

(a)

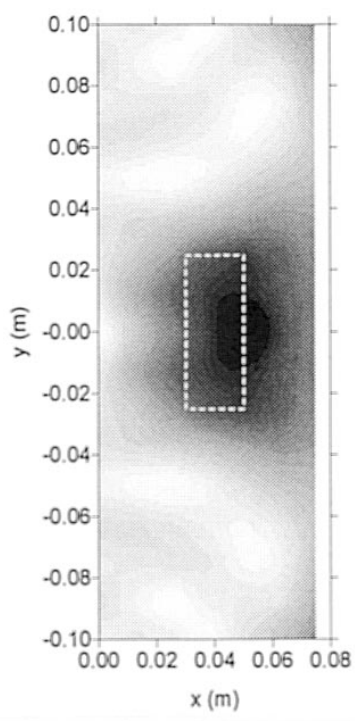

(c)

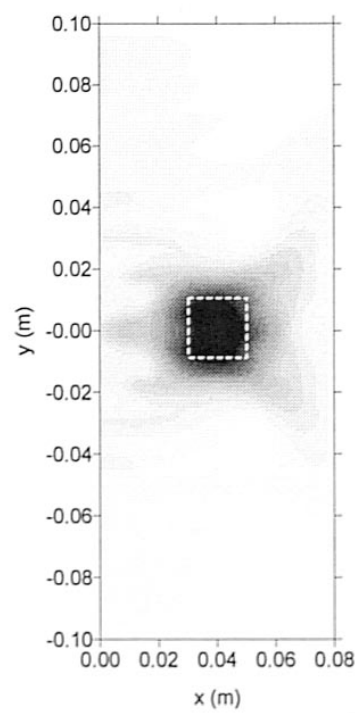

(b)

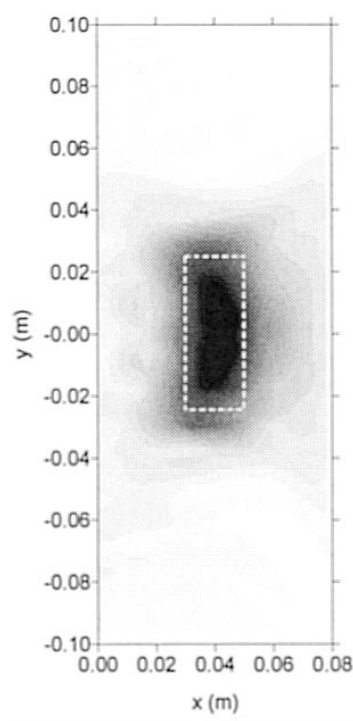

(d)

Fig. 10. Resolution improvement using higher frequency. (a) Case 5 using 5.2 GHz. (b) Case 5 using 10.0 GHz. (c) Case 6 using 5.2 GHz. (d) Case 6 using $10.0 \mathrm{GHz}$.

transmitting and the receiving arrays, and symmetric with respect to the diagonal in the measurement matrix. The calibration factors of each transmission measurement at $5.2 \mathrm{GHz}$ were used for calculating the calibrated measurement matrix in the experiments.

\section{B. Experimental Results}

A continuous $5.2 \mathrm{GHz}$ sinusoidal EM wave was generated from the signal analyzer and sent to the test specimen. Switch box controls the location of transmitting and receiving arrays from $S_{1,9}$ to $S_{8,16}$. Transmission measurements of each transmitting and receiving array pair at $5.2 \mathrm{GHz}$ were assembled into a measurement matrix.

Each measured signal was divided by the calibration factor at $5.2 \mathrm{GHz}$ as described in previous section, and was assem-

TABLE I

DESCRIPTIONS OF EXPERIMENTAL CASES

\begin{tabular}{cccc}
\hline No & Description & $\begin{array}{c}\text { Reconstructed } \\
\text { Area }(x \times y)\end{array}$ & $\begin{array}{c}\text { Exact } \\
\text { Locations of } \\
\text { Objects } \\
(x, y)(\mathrm{cm})\end{array}$ \\
\hline 1 & $\begin{array}{c}\text { Steel Sphere } \\
\text { At Center of } \\
\text { Concrete Panel }\end{array}$ & $4 \mathrm{~cm} \times 20 \mathrm{~cm}$ & $(0,0)$ \\
\hline 2 & $\begin{array}{c}\text { Rectangular Steel Bar } \\
\text { In the Air }\end{array}$ & $6 \mathrm{~cm} \times 20 \mathrm{~cm}$ & $(3,0)$ \\
\hline 3 & $\begin{array}{c}\text { Rectangular Steel Bar } \\
\text { In the Air }\end{array}$ & $6 \mathrm{~cm} \times 20 \mathrm{~cm}$ & $(3,-2)$ \\
\hline 4 & $\begin{array}{c}\text { Square Void } \\
(2 \mathrm{~cm} \times 2 \mathrm{~cm}) \\
\text { In Concrete }\end{array}$ & $8 \mathrm{~cm} \times 20 \mathrm{~cm}$ & $(4,0)$ \\
\hline 5 & $\begin{array}{c}\text { Rectangular Void } \\
(2 \mathrm{~cm} \times 5 \mathrm{~cm}) \\
\text { In Concrete }\end{array}$ & $8 \mathrm{~cm} \times 20 \mathrm{~cm}$ & $(4,0)$ \\
\hline
\end{tabular}

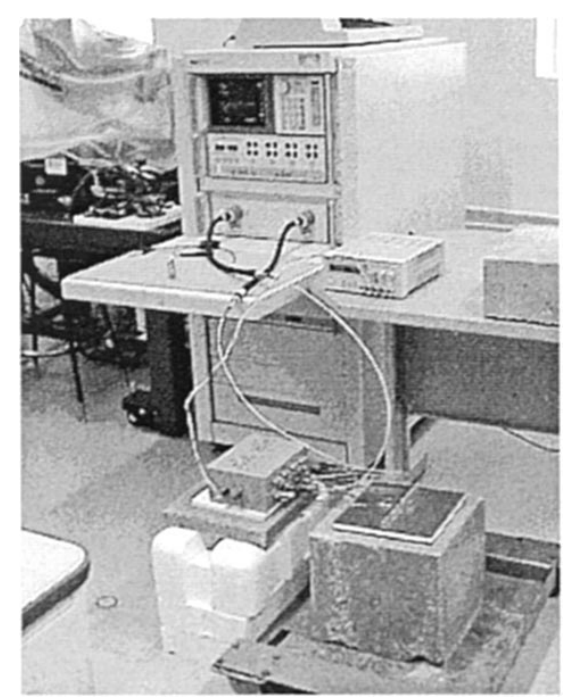

Fig. 11. Experimental setup.

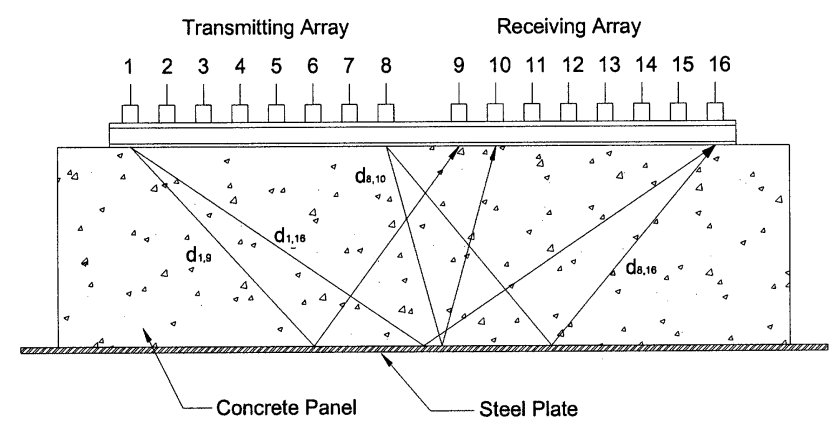

Fig. 12. Description of calibration scheme.

bled into the calibrated measurement matrix to be multiplied by numerical focusing operators. The reconstructed images of a center cut in each case are plotted in Fig. 13 by the amplitude of electric current distributions. All the images contain steel objects or air voids.

In case 1, the location of the steel sphere is detected as well as the size, although there are edge effects from the small size 

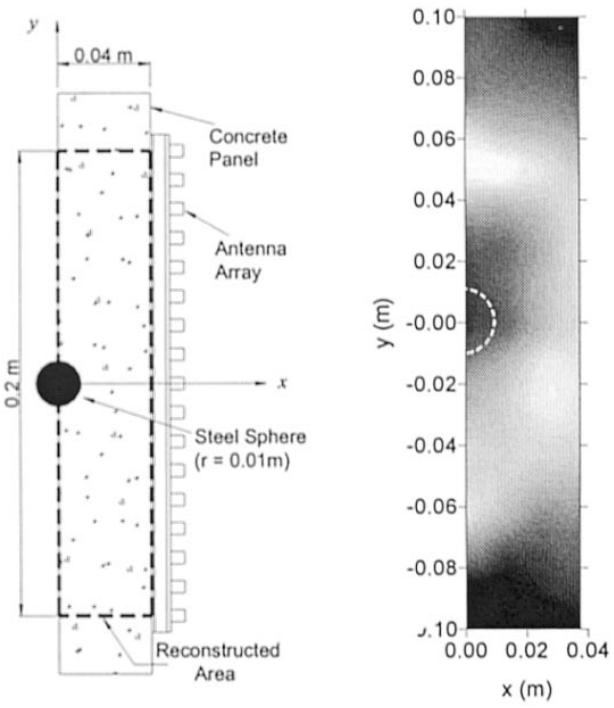

(a)
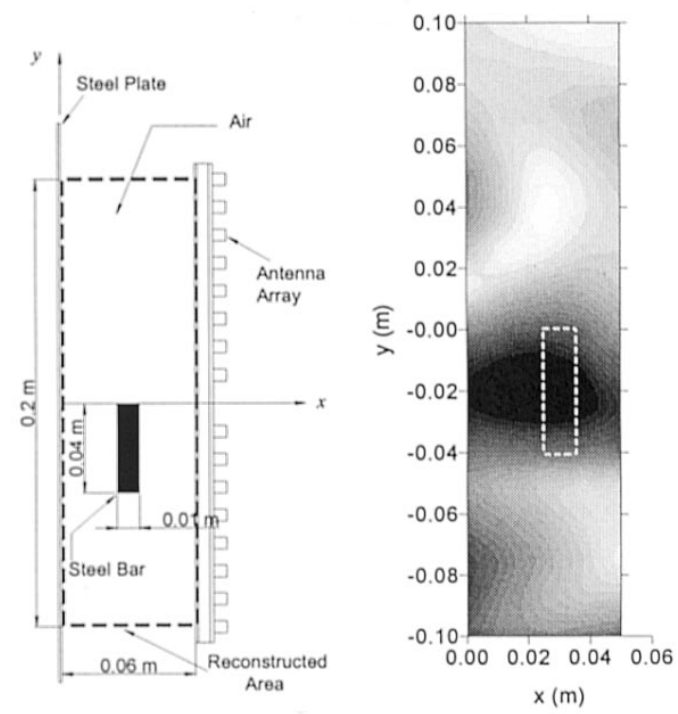

(c)

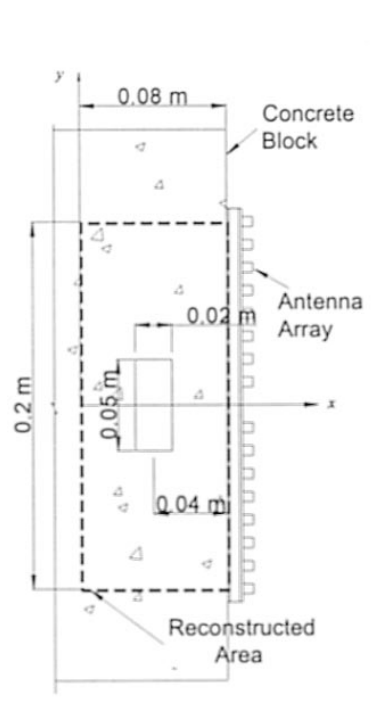

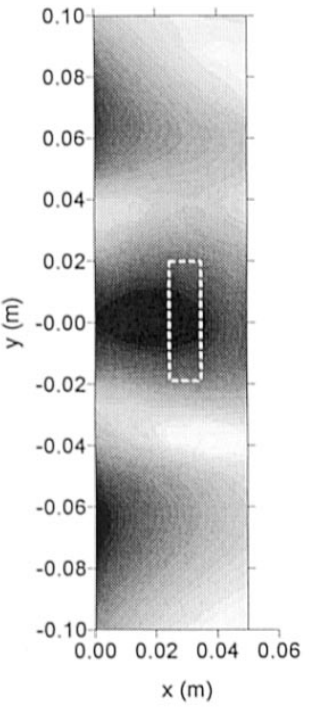

(b)

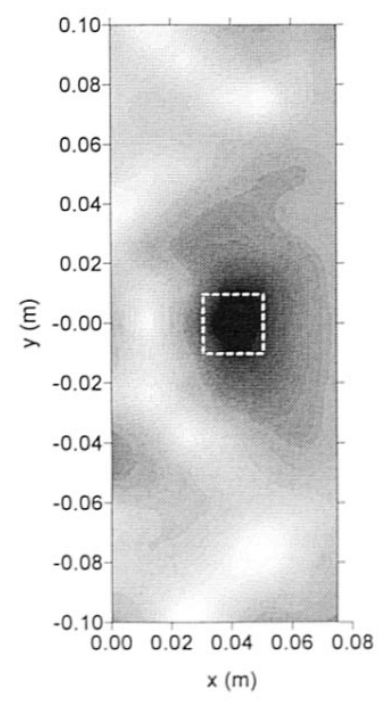

(d)
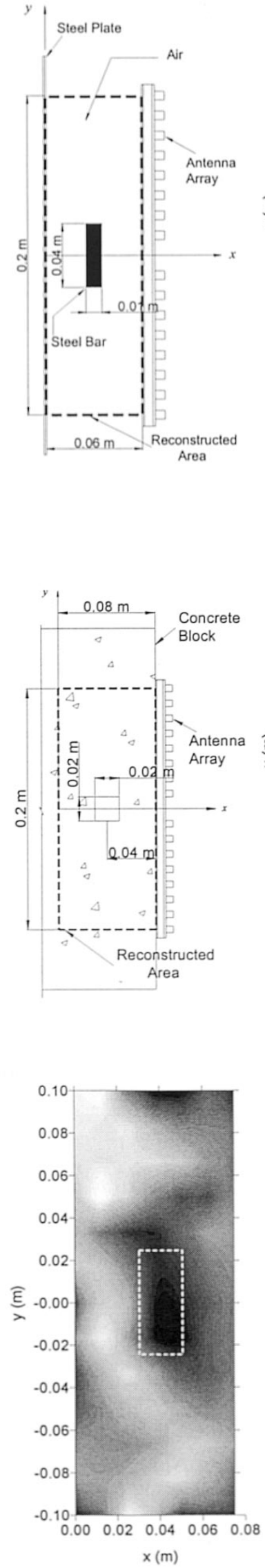

(e)

Fig. 13. Experimental results. (a) Case 1. (b) Case 2. (c) Case 3. (d) Case 4. (e) Case 5. 
of the concrete specimen, which add some noises to the result. Case 2 and case 3 are dealing with radiation into the air rather than concrete. As a result, the steel bars are detected by the location: reconstructed image in case 3 is shifted to the negative $y$ direction with respect to the image in case 2 , as expected. The shape of the steel bar could not be exactly reconstructed because of the lack of wave radiation into the air.

Case 4 and case 5 are more realistic cases, which demonstrate air voids inside concrete block. Two-dimensional descriptions are illustrated in Fig. 13(d) and (e). The results from both cases show that the images of square and rectangular Styrofoam's are successfully reconstructed in terms of size and location, as represented in Fig. 13(d) and (e). The resolution, which is $2.5 \mathrm{~cm}$ at $5.2 \mathrm{GHz}$, was not enough to reconstruct the exact size, which can be improved by using the higher frequency, as explained in the previous numerical simulation.

\section{CONCLUSION}

A subsurface focused microwave imaging technology was developed in this paper for detecting damages or objects inside concrete structures. The following conclusions can be drawn from simulation analysis and experiments.

1) An imaging reconstruction algorithm using a bifocusing operator was formulated for the subsurface imaging technology and verified through a series of numerical simulations. The results showed that the algorithm gives a uniformity of focusing intensity level and the resolution in the order of the wavelength in a dielectric medium (concrete in this case) can be achieved by focusing both in transmitting and receiving arrays.

2) A slot antenna array working in front of a dielectric medium was designed and fabricated. Through testing, it is found that the slot antenna array is appropriate to use for damage detection of concrete structures in terms of its radiation performance and bandwidth.

3) The effective focusing area of a planar antenna array is found to be a trapezoid, in which the focusing operator is effectively working.

4) From the results of the numerical simulation, steel or air objects within the effective focusing area can be successfully detected in terms of the location and the size. It was also verified that the resolution can be improved by increasing the illuminating frequency.

5) From the results of experiments using a concrete panel and concrete block, it is demonstrated that the steel objects and air void can be successfully detected in terms of the location in depth and the approximate size.

\section{REFERENCES}

[1] M. Q. Feng, F. De Flaviis, Y. J. Kim, and R. Diaz, “Application of electromagnetic waves in damage detection of concrete structures," in Proc. Int. Symp. Smart Structures and Materials, SPIE, Newport Beach, CA, Mar. 6-7, 2000, pp. 118-126.

[2] M. Q. Feng, F. De Flaviis, and Y. J. Kim, "Use of microwaves for damage detection of fiber reinforced polymer-wrapped concrete structures," $J$ Eng. Mechan., ASCE, vol. 128, no. 2, pp. 172-183, 2002.
[3] Y. J. Kim, F. De Flaviis, L. Jofre, and M. Q. Feng, "Microwave-based NDE of FRP-jacketed concrete structures," in Proc. 46th Int. SAMPE Symp., vol. 46, Long Beach, CA, May 6-10, 2001.

[4] — "Microwave cylindrical reflection imaging array for structural damage detection," in Proc. IEEE AP-S/URSI Symp., Boston, MA, June 2001, pp. 678-681.

[5] I. M. Gironés, L. Jofre, M. Ferrando, M. De Los Reyes, and J. C.J. Ch. Bolomey, "Microwave imaging with crossed linear arrays," Proc. Inst Elect. Eng., pt. H, vol. 134, pp. 249-252, June 1987.

[6] A. Broquetas, J. Romeu, J. M. Rius, A. Elias-Fuste, A. Cardama, and L. Jofre, "Cylindrical geometry: a further step in active microwave tomography," IEEE Trans. Microwave Theory Tech., vol. 39, pp. 836-844, May 1991.

[7] S. X. Pan and A. C. Kak, "A computational study of reconstruction algorithms for diffraction tomography: interpolation vs filtered-backpropagation," IEEE Trans. Acoust. Speech Signal Processing, vol. ASSP-31, pp. 1262-1275, Oct. 1983.

[8] R. Garg, P. Bhartia, I. Bahl, and A. Ittipiboon, Microstrip Antenna Design Handbook. Boston, MA: Artech House, 2001.

[9] C. Chen, W. E. McKinzie III, and N. G. Alexopoulos, "Stripeline-fed arbitrarily shaped printed-aperture antennas," IEEE Trans. Antennas Propagat., vol. 45, pp. 1186-1198, July 1997.

[10] C. A. Balanis, Advanced Engineering Electromagnetics. New York: Wiley, 1989.

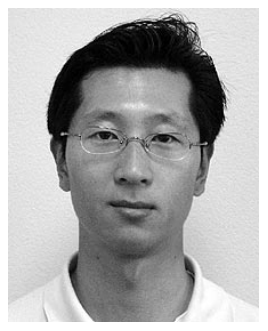

Yoo Jin Kim was born in Seoul, Korea, in 1972. He received the M.S. degree in civil engineering from the Korea Advanced Institute of Science and Technology (KAIST), Taejon, in 1998 and the Ph.D. degree, also in civil engineering, from the University of California, Irvine, in 2002.

From 1999 to 2002, he was Research Assistant with the Department of Civil and Environmental Engineering, University of California, Irvine, where he worked on development of microwave imaging technology using reflection tomographic antenna array. From 2002 to the present, he has been a Post Graduate Researcher, working on development of 3-D microwave imaging technology for condition assessment of civil infrastructures.

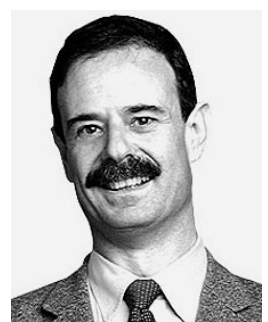

Luis Jofre (M'78) was born in Barcelona, Spain, in 1956. He received the M.Sc. (Ing) and Ph.D. (Doctor Ing.) degrees in electrical engineering (telecommunications engineering), from the Technical University of Catalonia (UPC), Barcelona, in 1978 and 1982, respectively.

From 1979 to 1980 , he was a Research Assistant with the Electrophysics Group, UPC, where he worked on the analysis and near-field measurement of antenna and scatterers. From 1981 to 1982, he was with the Ecole Superieure d'Electricite, Paris, France, where he was involved in microwave antenna design and imaging techniques for medical and industrial applications. In 1982, he was appointed Associate Professor with the Communications Department, Telecommunication Engineering School, UPC, where he became a Full Professor in 1989. From 1986 to 1987 , he was a Visiting Fulbright Scholar at the Georgia Institute of Technology, Atlanta, working on antennas, and electromagnetic imaging and visualization. From 1989 to 1994, he served as Director of the Telecommunication Engineering School (UPC), and from 1994 to 2000, he was UPC Vice-rector for Academic Planning. His research interests include antennas, scattering, electromagnetic imaging, and wireless communications. He has published more than 100 scientific and technical papers, reports, and chapters in specialized volumes. During 2000 and 2001, he was a Visiting Professor with the Electrical and Computer Engineering Department, Henry Samueli School of Engineering, University of California, Irvine, where he focused on antennas and systems miniaturization for wireless and sensing applications. 


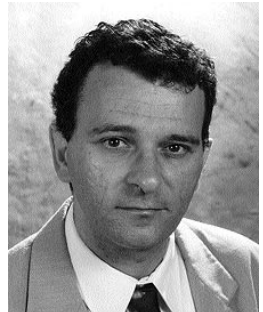

Franco De Flaviis (M'98) was born in Teramo, Italy, in 1963. He received the Laurea degree in electronics engineering from the University of Ancona, Italy, in 1990. He received the M.S. and Ph.D. degrees in electrical engineering from the Department of Electrical Engineering, University of California at Los Angeles (UCLA), in 1994 and 1997, respectively.

In 1991, he was an engineer with Alcatel, working as as researcher specialized in the area of microwave mixer design. In 1992, he was a Visiting Researcher with UCLA working on low intermodulation mixers. Currently, he is an Assistant Professor with the Department of Electrical and Computer Engineering, University of California, Irvine. His research interests are in the field of computer-aided electromagnetics for high-speed digital circuits and antennas, and microelectromechanical switches (MEMS) for RF applications fabricated on unconventional substrates such as printed circuit board and microwave laminates.

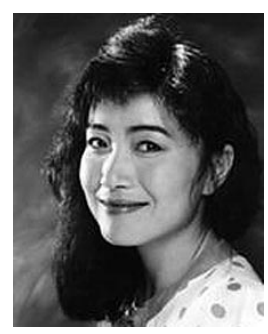

Maria Q. Feng received the Ph.D. degree in mechanical engineering from the University of Tokyo, Tokyo, Japan.

She joined the University of California, Irvine, in 1992, where she is a Professor of civil engineering and Leader of the Environmental and Civil Infrastructure Layer of the California Institute of Telecommunications and Information Technology. Her research interests are primarily in the fields of innovative and interdisciplinary science and technology for earthquake and wind protection, sensors and health monitoring, and damage detection of civil infrastructure systems. Her notable achievements include the development of an intelligent friction controllable sliding isolation system for seismic protection of buildings, unique optical fiber sensors for measuring dynamic response of civil engineering structures, and microwave imaging technology for nondestructive evaluation of internal and invisible damage in concrete structures.

Dr. Feng has received numerous awards and recognition including the CAREER Award in 1995 from the National Science Foundation, and the 1995 Alfred Noble Prize, the 1995 Collingwood Prize, the 1997 Charles Pankow Finalist Award for Innovation, and the 1999 Walter L. Huber Civil Engineering Research Prize, all from the American Society of Civil Engineers. 